\title{
Bilinear Discriminant Analysis Hashing: A Supervised Hashing Approach for High-Dimensional Data
}

\author{
Yanzhen Liu ${ }^{1}$, Xiao Bai ${ }^{1}$, Cheng Yan $^{1}$, and Jun Zhou ${ }^{2}$ \\ ${ }^{1}$ School of Computer Science and Engineering, Beihang University, China \\ $\{$ lyzeva, baixiao, beihangyc\}@buaa.edu.cn \\ ${ }^{2}$ School of Information and Communication Technology, Griffith University, Australia \\ jun.zhou@griffith.edu.au
}

\begin{abstract}
High-dimensional descriptors have been widely used in object recognition and image classification. How to quickly index highdimensional data into binary codes is a challenging task which has attracted the attention of many researchers. Most existing hashing solutions for high-dimensional dataests are based on unsupervised schemes. On the other hand, existing supervised hashing methods cannot work well on high-dimensional datasets, as they consume too much time and memory to index high-dimensional data. In this paper, we propose a supervised hashing method Bilinear Discriminant Analysis Hashing (B$\mathrm{DAH})$ to solve this problem. BDAH leverages supervised information according to the idea of Linear Discriminant Analysis (LDA), but adopts bilinear projection method. Bilinear projection needs two small matrices rather than one big matrix to project data so that the coding time and memory consumption are drastically reduced. We validate the proposed method on three datasets, and compare it to several state-of-the-art hashing schemes. The results show that our method can achieve comparable accuracy to the state-of-the-art supervised hashing schemes, while, however, cost much less time and memory. What's more, our method outperforms unsupervised hashing methods in accuracy while achieving comparable time and memory consumption.
\end{abstract}

\section{Introduction}

Nearest Neighbor (NN) search is a basic and important step in image retrieval. For a large scale dataset of size $n$, the complexity of NN search $O(n)$ is too high for big data processing. To solve this problem, approximate nearest neighbor(ANN) methods have been proposed, in which hashing is a class of wellbehaved methods. The basic idea of hashing is to use binary code to represent high-dimensional data, with similar data pairs having smaller Hamming distance of binary codes. Then the task of finding the nearest neighbors of a data point can be transformed into finding the most similar hash codes. With hashing, we only need to apply XOR operations between binary hash codes, rather than calculating the Euclidean distances between data vectors which involves frequent addition and multiplication operations. 
To calculate proper hash codes, we should construct a mapping from highdimension Euclidean space to Hamming space and preserve the original Euclidean distance of data in the Hamming space. There are already many works on hash code learning. According to leveraging supervised information or not, hashing methods can be classified into supervised hashing methods [1-7] or unsupervised hashing methods [8-15]. Hashing methods can also be divided into data-dependent $[9,2,11,4-6,16,17]$ and data-independent $[8,12,13]$ depending on whether to use training data to generate hash codes or not.

A large number of hashing methods are based on linear projection and 0-1 quantization. A simple and basic data-independent method is Locality Sensitive Hashing(LSH) [8]. LSH is based on generating random linear projection vectors in the Euclidean space. After projection on one vector, data are quantized into 0 or 1 by its sign, consequently generating one bit of hash code. This method can also be comprehended as hyperplane partitioning the Euclidean space. A hyperplane correspond to one projection vector and data points on one side of the hyperplane are coded 0 , while at the other side are coded 1 . LSH is easy to implement and successfully reduces the NN search to sublinear query time with acceptable accuracy. However, because the hyperplanes are randomly set and not depend on the dataset, we need longer codes to achieve accuracy. To overcome this shortcoming, many data-dependent methods have been proposed, such as SSH [2] and ITQ [11], which try to learn projection from the distribution of the dataset. They achieved higher accuracy than LSH.

In projection based hashing schemes, dimension of projection vector is the same as the dimension of the original data. Nowadays, features of thousands of dimensions appear with high retrieval and classification accuracy, such as Fisher Vectors (FV) [18], Vectors of Locally Aggregated Descriptors (VLAD) [19] and Deep Neural Networks Features (DNN) [20]. Projection vectors for these data are also high-dimensional. What's more, hash codes of these high-dimensional datasets are also longer to ensure accuracy. As a consequence, the projection matrix is huge for high-dimensional dataset, and more time is needed to calculate hash codes as well as train the projection matrix. Several works are concentrating on learning hash codes for high-dimensional data [21-23]. However, they are all unsupervised methods without utilizing supervised information, and supervised hashing scheme for high-dimensional datasets has seldom be studied. Existing supervised hashing schemes like Kernel-Based Supervised Hashing(KSH) [5] and Supervised Discrete Hashing(SDH) [6] will cost too much time and memory to calculate hash codes. They are unfit for high-dimensional datasets.

In this papaer, we propose a supervised hashing scheme specific for highdimensional datasets, and we name it Bilinear Discriminant Analysis Hashing $(\mathrm{BDAH})$. In the dimension reduction part, we utilize a bilinear projection model from 2DLDA [24] to minimize within-class distance and maximize between-class distance of projected data. According to 2DLDA, we resize long 1-D vectors into 2-D matrices and use two projection matrices to project the 2-D matrices. In this way, although the number of matrices increases, total elements in the projection matrices are drastically reduced, and meanwhile the time and memory consump- 
tion for hash code calculation is reduced. Then we optimize the two projection matrices iteratively. After projecting the data, we quantize the projected vectors into binary codes. To minimize quantization loss, Iterative Quantization [11] is adopted to calculate an optimal rotation matrix.

There are three contributions in this paper. Firstly, BDAH projects data in 2-D form, so it can better protect the inner structure of 2-D form descriptors such as LLC [25] and VLAD [19]. Secondly, bilinear projection drastically reduces time and memory cost for hash code generation, so it is suitable for hashing on highdimensional dataset. Finally, our BDAH is a supervised hashing scheme, and the utilization of label information help to get better retrieval accuracy of the hash codes than unsupervised hashing schemes. We validate the effectiveness of our BDAH on 3 datasets: AwA [26], MNIST [27] and ILSVRC2010 [28], comparing to CCA-ITQ [11], KSH [5], BPBC [21], CBE-opt [22] and PCA-ITQ [11]. The experiments show that our method reduces time and memory consumption while keeping comparable accuracy to other state-of-the-art hashing schemes.

The rest of this paper is organized as follows. Section 2 introduces related work on high-dimensional data hashing. Section 3 describes the technical process of BDAH. Section 4 introduces our experiments and the performances of our proposed methods. Finally, conclusions are drawn in Section 5 .

\section{Related Work}

\subsection{Hashing Schemes for High-dimensional Dataset}

Recently, several works have been reported which aim at accelerating highdimensional projection process in different ways [21-23]. The essence of these method is to reduce the actual number of variables in the projection matrix. However they are all unsupervised hashing schemes and cannot leverage supervised information, so they can't achieve particularly high performance.

Bilinear Projection based Binary Codes(BPBC) [21] reshapes the data vectors into matrices, and learns the projection matrix (orthogonal projection matrix) with the 2-D data form. Then BPBC can use two small projection matrices instead of one big projection matrix. In this way, the size of rotation matrix decreases from $n^{2}$ to $n_{1}^{2}+n_{2}^{2}\left(n_{1}+n_{2}=n\right)$.

Circulant Binary Embedding(CBE) [22] assumes that the rotation matrix has circulant inner structure and transforms the linear projection into form of circulant convolution. The elements in the projection matrix of $\mathrm{CBE}$ is constructed by one of the row vectors in the matrix, so the storage of projection matrix reduces to the size of a vector. Then it uses FFT to speed up the coding process. For code training, CBE adds a regularizer in the quantization loss function to make the rotation matrix approximate orthogonal matrix. CBE is quicker than $\mathrm{BPBC}$ in hash coding.

Sparse Projections approach (SP) [23] adds a sparse regularizer to the projection matrix in the quantization loss function. The sparse projection matrix reduces the redundant information in the high-dimensional projection matrix, so SP can achieve comparable accuracy to ITQ but has much less encoding time. 


\subsection{Linear Discriminant Analysis}

For a $d$-dimensional training set $X=\left\{\boldsymbol{x}_{1}, \boldsymbol{x}_{2}, \ldots, \boldsymbol{x}_{n}\right\}$ belonging to $c$ differen$\mathrm{t}$ classes $\Pi_{1}, \Pi_{2}, \ldots, \Pi_{c}$, LDA [29] tries to project the data points into a $b$ dimensional space with a linear projection matrix $W \in \mathbb{R}^{d \times b}$, in which data points in the same class become nearer while data points in different classes becomes farther. Suppose there are $a_{i}$ different data points in class $\Pi_{i}$, we get our objective formulation

$$
J_{L}(W)=\max \left(D_{w}\right)^{-1} D_{b},
$$

where

$$
D_{w}=\sum_{i=1}^{c} \sum_{j \in \Pi_{i}}\left\|W^{T} \boldsymbol{x}_{j}-W^{T} \boldsymbol{m}_{i}\right\|_{F}^{2}
$$

is the within-class distance, and

$$
D_{b}=\sum_{i=1}^{c} a_{i}\left\|W^{T}\left(\boldsymbol{m}_{i}-\boldsymbol{m}_{0}\right)\right\|_{F}^{2}
$$

is the between-class distance.

Eq.(1) can be further derived into

$$
J_{L}(W)=\max \operatorname{trace}\left(\left(W^{T} S_{w} W\right)^{-1} W^{T} S_{b} W\right) .
$$

This optimization problem is equivalent to the generalized eigenvalue problem $S_{b} \boldsymbol{w}_{i}=\lambda_{i} S_{w} \boldsymbol{w}_{i}$, where $\lambda_{i}$ denotes the $i$ th largest eigenvalue and $\boldsymbol{w}_{i}$ is its corresponding eigenvector as well as the $i$ th column of the projection matrix $W$. We want the projected space to be $b$-dimensional, so we pick $b$ eigenvectors.

\section{$3 \quad$ Bilinear Discriminant Analysis Hashing}

For a $d$-dimensional training set $\left\{\boldsymbol{x}_{1}, \boldsymbol{x}_{2}, \ldots, \boldsymbol{x}_{n}\right\}$ having $c$ classes $\Pi_{1}, \Pi_{2}, \ldots, \Pi_{c}$, we want to map the data vectors into $b$-bit hash codes $\left\{\boldsymbol{b}_{1}, \boldsymbol{b}_{2}, \ldots, \boldsymbol{b}_{n}\right\}$, where data in the same class have smaller hamming distances while data in different classes having larger hamming distances. It is too hard for us to compute the projection directly. So we relax the problem into two steps. Firstly, we project the data vectors into a lower $b$-dimensional vectors $\left\{\boldsymbol{y}_{1}, \boldsymbol{y}_{2}, \ldots, \boldsymbol{y}_{n}\right\}$ to make withinclass distance smaller and between-class distance higher (Sec. 3.1). Secondly, we quantize the $b$-dimensional data into binary codes (Sec. 3.2).

\subsection{Learning 2DLDA Projection Matrices}

When there is a need to project a data vector $\boldsymbol{x} \in \mathbb{R}^{d}$ to a lower $b$-dimensional space, people often use a matrix $W \in \mathbb{R}^{d \times b}$ to multiply the data vector $\boldsymbol{x}$, and get the low-dimensional projected vector $\boldsymbol{y} \in \mathbb{R}^{b}$ :

$$
\boldsymbol{y}=W^{T} \boldsymbol{x} .
$$


If the dimension of the data $d$ is very high, the projection matrix $W$ will be very large as well.

In our method, we adopt another projection form [24]. Firstly, we need to express data vector $\boldsymbol{x}$ in a matrix form. Then the dataset changes from a vector set into a matrix set $\left\{A_{1}, A_{2}, \ldots, A_{n}\right\} \in \mathbb{R} d_{1} \times d_{2}$. The elements in matrix $A_{i}$ are the same as the elements in vector $\boldsymbol{x}_{i}$, so $d=d_{1} \times d_{2}$. And $\boldsymbol{x}=\operatorname{vec}(A)$ where $\operatorname{vec}(A)$ denotes converting the matrix $A$ into a vector by concatenating the columns of $A$.

For the matrix set $\left\{A_{1}, A_{2}, \ldots, A_{n}\right\}$, the projection can change into the following form

$$
\boldsymbol{y}=\operatorname{vec}\left(L^{T} A R\right) .
$$

In Eq.(6), $L \in \mathbb{R}^{d_{1} \times b_{1}}$ and $R \in \mathbb{R}^{d_{2} \times b_{2}}$ are the projection matrices, where $b=$ $b_{1} \times b_{2}$. We call it bilinear projection.

It is easy to prove that

$$
\operatorname{vec}\left(L^{T} A R\right)=\left(R^{T} \otimes L^{T}\right) \operatorname{vec}(A)=\hat{W}^{T} \boldsymbol{x},
$$

where $\otimes$ denotes the Kronecker product. So the bilinear projection with two projection matrices is equivalent to common projection with one matrix.

In common projection method, the projection matrix $W$ have $d * b=\left(d_{1} d_{2}\right) *$ $\left(b_{1} b_{2}\right)=d_{1} b_{1} * d_{2} b_{2}$ elements, the complexity of projection is $O\left(d_{1} d_{2}\right)$. But in bilinear projection method, two projection matrices $L$ and $R$ have $d_{1} b_{1}+$ $d_{2} b_{2}$ elements in total, and the bilinear projection procedure has $O\left(d_{1}^{2} d_{2}+d_{2}^{2}\right)$ time complexity. We can see that both the time and memory complexity of the projection procedure are drastically reduced.

With two bilinear projection matrices, the between-class distance $D_{b}$ and within-class distance $D_{w}$ in LDA turn into the following form

$$
\begin{gathered}
D_{b}=\sum_{i=1}^{c} a_{i}\left\|L^{T}\left(M_{i}-M_{0}\right) R\right\|_{F}^{2}, \\
D_{w}=\sum_{i=1}^{c} \sum_{A \in \Pi_{i}}\left\|L^{T}\left(A-M_{i}\right) R\right\|_{F}^{2},
\end{gathered}
$$

where

$$
M_{i}=\frac{\sum_{j \in \Pi_{i}} A_{j}}{n}(1 \leqslant i \leqslant c)
$$

and

$$
M_{0}=\operatorname{frac} \sum_{i=1}^{n} A_{i} n .
$$

$D_{b}$ and $D_{w}$ can be further derived into the following form

$$
D_{b}=\operatorname{trace}\left(\sum_{i=1}^{c} a_{i} L^{T}\left(M_{i}-M_{0}\right) R R^{T}\left(M_{i}-M_{0}\right)^{T} L\right),
$$




$$
D_{w}=\operatorname{trace}\left(\sum_{i=1}^{c} \sum_{A \in \Pi_{i}} L^{T}\left(A-M_{i}\right) R R^{T}\left(A-M_{i}\right)^{T} L\right) .
$$

We want to maximize the between-class distance $D_{b}$ while minimize the within-class distance $D_{w}$. That is to maximize $\mathrm{J}(\mathrm{L}, \mathrm{R})$ given below.

$$
J(L, R)=\max \operatorname{trace}\left(\left(D_{w}\right)^{-1} D_{b}\right)
$$

It is difficult to optimize $L$ and $R$ simultaneously. So we optimize $L$ and $R$ iteratively.

Fix $R$ and update $L$.

With fixed $R$, we can derive our object function $J(L)$

$$
\left.J_{L}(L)=\max \operatorname{trace}\left(\left(L^{T} S_{w}^{R} L\right)^{-1}\left(L^{T} S_{b}^{R} L\right)\right)=\max \operatorname{trace}\left(S_{w}^{R}\right)^{-1} S_{b}^{R}\right),
$$

where

$$
\begin{aligned}
S_{b}^{R} & =\sum_{i=1}^{c} a_{i}\left(M_{i}-M_{0}\right) R R^{T}\left(M_{i}-M_{0}\right)^{T}, \\
S_{w}^{R} & =\sum_{i=1}^{c} \sum_{A \in \Pi_{i}}\left(A-M_{i}\right) R R^{T}\left(A-M_{i}\right)^{T} .
\end{aligned}
$$

Then we get the optimized $L$ by doing eigen decomposition on the matrix $\left(S_{w}^{R}\right)^{-1} S_{b}^{R}$.

Fix $L$ and update $R$.

Analogously, with fixed $L$, we can derive our object function $J(R)$

$$
J(R)=\max \operatorname{trace}\left(\left(R^{T} S_{w}^{L} R\right)^{-1}\left(R^{T} S_{b}^{L} R\right)\right),
$$

where

$$
\begin{aligned}
S_{b}^{L} & =\sum_{i=1}^{c} a_{i}\left(M_{i}-M_{0}\right)^{T} L L^{T}\left(M_{i}-M_{0}\right), \\
S_{w}^{L} & =\sum_{i=1}^{c} \sum_{A \in \Pi_{i}}\left(A-M_{i}\right)^{T} L L^{T}\left(A-M_{i}\right) .
\end{aligned}
$$

The iteration stops after convergence and we got the locally optimized $L$ and $R$.

\subsection{Quantization into Binary Codes}

After the 2DLDA projection $L$ and $R$ are recovered, we can calculate tje projected vectors $\boldsymbol{y}_{i}=\operatorname{vec}\left(L^{T} A_{i} R\right)$. The low-dimensional dataset is denoted by

$$
Y=\left[\boldsymbol{y}_{1}, \boldsymbol{y}_{2}, \ldots, \boldsymbol{y}_{n}\right]^{T} \text {. }
$$

Next, we try to quantize $Y$ into binary codes $B \in\{1,-1\}^{n \times b}$. The most direct method is just using the sign function. That is

$$
B=\operatorname{sign}(Y) \text {. }
$$


Direct quantization may cause great loss as explained in [11]. So we try to calculate an optimized rotation matrix $U \in \mathbb{R}^{b \times b}$ to minimize the quantization loss $Q$.

$$
\begin{aligned}
& Q=\|B-Y U\|_{F} \\
& U U^{T}=I
\end{aligned}
$$

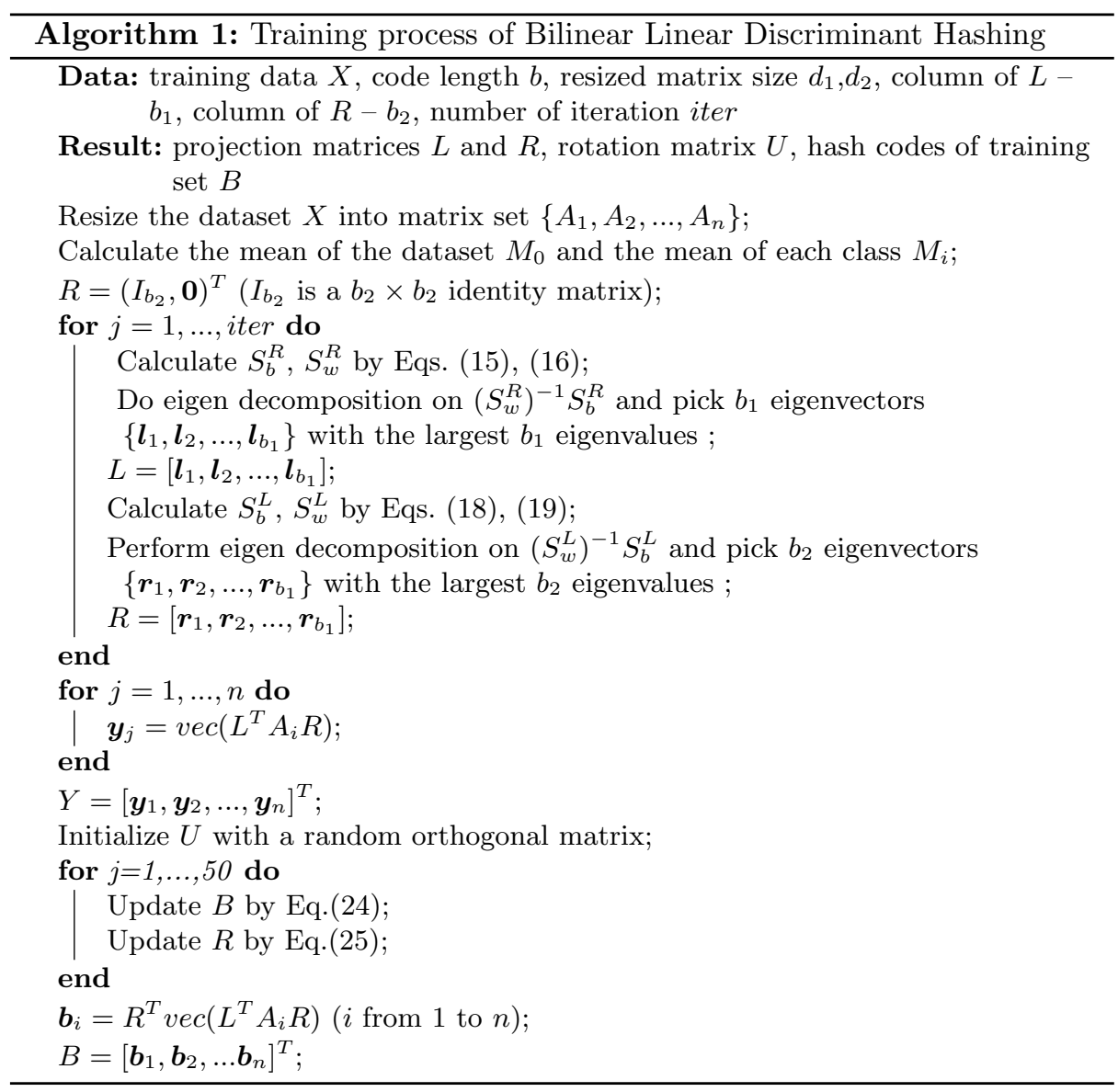

And the solution is like the iterative process in [11]. We do the iterations between $B$ and $U$ for several times and get a locally optimal solution.

Fix $U$ and update $B$.

For a fixed $U$, we can further derive Eq.(22) into

$$
\begin{aligned}
Q & =\|B\|_{F}^{2}+\|Y\|_{F}^{2}-2 \operatorname{tr}\left(B U^{T} Y^{T}\right) \\
& =-2 \operatorname{tr}\left(B U^{T} Y^{T}\right)+\text { const. }
\end{aligned}
$$


Because $B$ and $Y$ is fixed, Eq.(23) is equivalent to

$$
\max \operatorname{tr}\left(B U^{T} Y^{T}\right) .
$$

And we can derive the optimal $B$ from

$$
B=\operatorname{sign}(Y U) .
$$

Fix $B$ and update $U$.

For a fixed $B$, Eq.(22) becomes the classical Orthogonal Procrustes Problem [30]. Compute SVD of the matrix $B^{T} Y=S \Omega \hat{S}^{T}$ and we get the optimal $U$ by

$$
U=\hat{S} S^{T}
$$

After the $Q$ converges, we get the locally optimal solution of Eq. (22). Here 50 iterations of optimization are adopted, which is enough to achieve convergence [11].

The above is the training process of our BLDH. Algorithm 1 is the pseudocode on training of BLDH. Through training, we get the bilinear projection matrice $L, R$ and rotation matrix $U$. For a new query $\boldsymbol{q}$, we can get its hash code by first resize it to a $d_{1} \times d_{2}$ matrix $Q_{q}$ and then calculate by the equation

$$
\boldsymbol{b}=\operatorname{sign}\left(U^{T} \operatorname{vec}\left(L^{T} Q_{q} R\right)\right) .
$$

\subsection{Addition LDA Projection for Further Accuracy}

To achieve further accuracy of our hash code, we can project the data once more with LDA after the bilinear projection of 2DLDA [24]. For a $d$-dimensional dataset $\left\{\boldsymbol{x}_{i}\right\}(i=1 \ldots . n)$, we want to get $b$-bit hash code. We can first project the data into $b 1 * b 2$-dimensional vectors $\left\{\boldsymbol{y}_{i}\right\}(i=1 \ldots n)$ by bilinear projection of 2DLDA (Sec. 2.2), where $b 1 * b 2=b$. Then we project $\left\{\boldsymbol{y}_{i}\right\}(i=1 \ldots n)$ into $b$-dimensional vector $\left\{\boldsymbol{z}_{i}\right\}(i=1 \ldots n)$ by LDA (Sec. 2.2). Finally, we quantize $\left\{\boldsymbol{z}_{i}\right\}(i=1 \ldots n)$ into binary code (Sec. 3.2). In this paper, we denote this hashing scheme with 2-step projection $\mathrm{BDAH}^{0}$. Experiments show that, combined with $\mathrm{LDA}, \mathrm{BDAH}^{0}$ can achieve much better retrieval accuracy.

\section{Experiment}

To test the effectiveness of our method, we conducted experiments on 3 datasets: AwA [26], CIFAR-10 [31] and ILSVRC2010 [28] . Our BDAH and BDAH ${ }^{0}$ were compared against several state-of-the-art methods including two supervised method CCA-ITQ [11] and KSH [5], and three unsupervised methods PCAITQ [11], BPBC [21] and CBE-opt [22]. We used the publicly available codes of these methods. All our experiments were run on a PC with a $3.5 \mathrm{GHz}$ Intel Core CPU and 32GB RAM. 


\begin{tabular}{|r|r|r|r|r|r|}
\hline Methods & 16bits & 36bits & 64bits & 100bits & 256bits \\
\hline BDAH $^{0}$ & 16 & 29 & 46 & 67 & 152 \\
\hline BDAH & 6 & 16 & 40 & 88 & 528 \\
\hline BPBC & 4 & 6 & 8 & 10 & 16 \\
\hline KSH & 9640 & 9687 & 9752 & 9837 & 10202 \\
\hline CBE-opt & 64 & 64 & 64 & 64 & 64 \\
\hline PCA-ITQ & 512 & 1152 & 2048 & 3200 & 8192 \\
\hline CCA-ITQ & 512 & 1152 & 2048 & 3200 & 8192 \\
\hline
\end{tabular}

Table 1. Memory consumption (KB) to hash new data into binary codes on AwA datset.

\subsection{MNIST: Retrieval with Raw Image}

The MNIST [27] dataset has $6000028^{*} 28$ small greyscale images of handwritten digits from ' 0 ' to '9'. Each small image is represented by a 784-dimensional vector and a single digit label. Each 784-dimensional vector is stretched from a corresponding $28^{*} 28$ greyscale image. We used 54000 random images as the training set, and 6000 random images as the query samples.

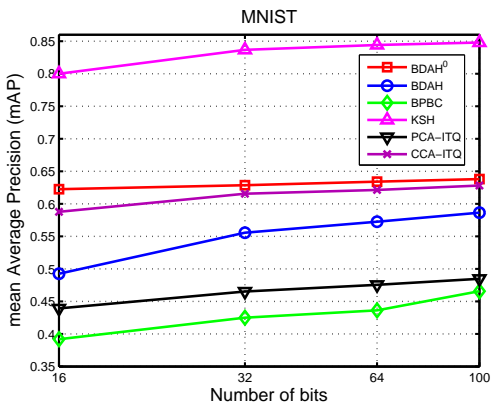

(a) mAP for different code bits.

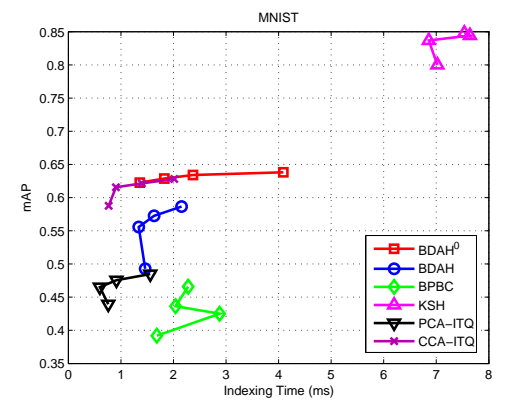

(b) Coding time vs. mAP for different code bits.

Fig. 1. Results on the MNIST dataset.

We set the hash code length to 16bits, 36bis, 64bits and 100bits in this dataset. Figure 1 reports the results on this dataset. Table 2 shows the training time and coding time of each method. In supervised methods, our BDAH ${ }^{0}$ exceeds CCA-ITQ in mAP, but needs a little more time on binary coding. BDAH ${ }^{0}$ has lower mAP than KSH, but consumes much less time than KSH. For unsupervised methods, $\mathrm{BDAH}^{0}$ and $\mathrm{BDAH}$ achieve comparable time consumption to them while having much higher mAP. 


\begin{tabular}{|c|c|r|r|r|r|}
\hline \multicolumn{2}{|c|}{ Methods $^{\text {16bits }}$} & 25bits & 64bits & 100bits \\
\hline \multirow{2}{*}{ BDAH $^{0}$} & Training & 8254.40 & 9373.46 & 9930.19 & 11624.55 \\
\cline { 2 - 6 } & Test & 4.18 & 4.95 & 5.03 & 6.31 \\
\hline \multirow{2}{*}{ BDAH $^{0}$} & Training & 8541.15 & 9172.20 & 9463.68 & 10156.50 \\
\cline { 2 - 6 } & Test & 3.93 & 5.95 & 5.28 & 6.70 \\
\hline \multirow{2}{*}{ BDAH } & Training & 8026.10 & 8865.57 & 9219.09 & 10324.81 \\
\cline { 2 - 6 } & Test & 3.42 & 3.88 & 4.47 & 5.34 \\
\hline \multirow{2}{*}{ KSH } & Training & 38055.16 & 72090.40 & 128932.07 & 199796.28 \\
\cline { 2 - 6 } & Test & 16.70 & 17.67 & 16.76 & 17.41 \\
\hline \multirow{2}{*}{ CCA-ITQ } & Training & 625.52 & 1126.64 & 1901.62 & 3211.55 \\
\cline { 2 - 6 } & Test & 1.74 & 2.10 & 3.86 & 4.98 \\
\hline \multirow{2}{*}{ PCA-ITQ } & Training & 441.86 & 669.27 & 1077.90 & 1815.74 \\
\cline { 2 - 6 } & Test & 1.02 & 1.26 & 2.08 & 3.34 \\
\hline
\end{tabular}

Table 2. Time consumption (milliseconds) of different methods on the MNIST dataset.

\subsection{AwA: Retrieval with DNN Features}

Animals with Attributes (AwA) [26] is a dataset with different kinds of animals. It has 30475 animal images belonging to 50 animal classes. In the experiments, we used a subset containing 9460 images. A seven-layer CaffeNet [20] was adopted to extract 4096-dimensional feature from AwA. The 4096-dimensional output of the fully-connected layer was used as our feature to calculate hash codes. In our $\mathrm{BDAH}^{0}$ and BDAH methods and BPBC in [21], we resized every DNN-4096 feature into a $64^{*} 64$ matrix and then used the matrices to generate hash codes in our retrieval experiment.

We tested all methods on AwA with 16bits, 36bits, 64bits, 100bits and 256bits codes. The memory consumption of indexing is reported on Table 1. For BDAH ${ }^{0}$, $\mathrm{BDAH}$ and BPBC, memory consumption is bilinear projection matrices, and for PCA-ITQ and CCA-ITQ, is the full projection matrix. KSH needs to store several anchor points and a kernel projection matrix, thus acquiring more memory. CBE-opt has the least memory consumption because it only needs to store a vector, rather than matrices in other methods. We can see clearly that, our $\mathrm{BDAH}^{0}$ and BDAH require less memory than PCA-ITQ and CCA-ITQ. The longer codes we needs, the more memory consumption $\mathrm{BDAH}^{0}$ and $\mathrm{BDAH}$ save. Figure 2 shows the P-R curves and time vs. mAP for the above-mentioned methods on AwA and Table 3 shows the training time and coding time of each method. $\mathrm{BDAH}^{0}$ still shows good balance in accuracy and time consumption. KSH is still best in mAP, but worst in indexing time than all the other methods. CCA-ITQ has the minimum coding time while PCA-ITQ trains faster, but both PCA-ITQ and CCA-ITQ cannot achieve as good accuracy as BDAH ${ }^{0}$.

\subsection{ILSVRC2010: Retrieval with VLAD Features}

ILSVRC2010 is a subset of ImageNet [28]. ImageNet is a hierarchical image dataset organized following the hierarchy of WordNet [32]. In our experiments, 


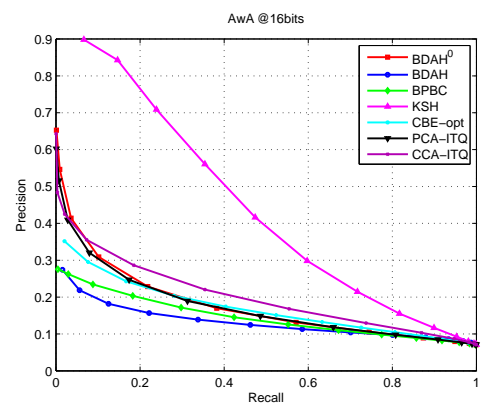

(a) P-R curves @16-bits

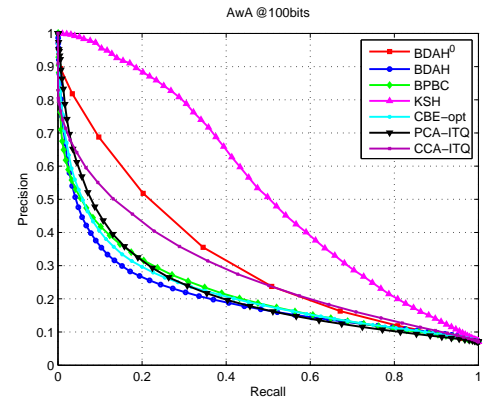

(c) P-R curves @100-bits

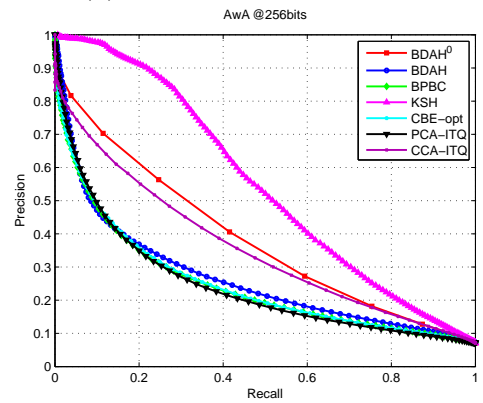

(e) P-R curves @256-bits

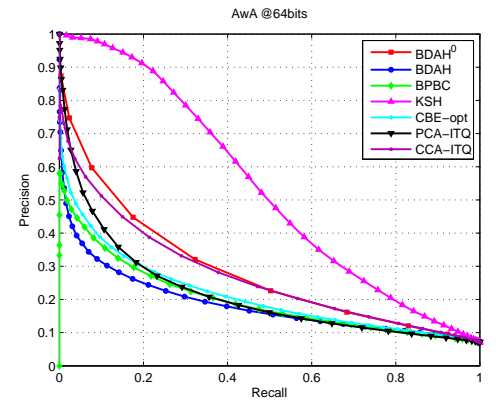

(b) P-R curves @64-bits

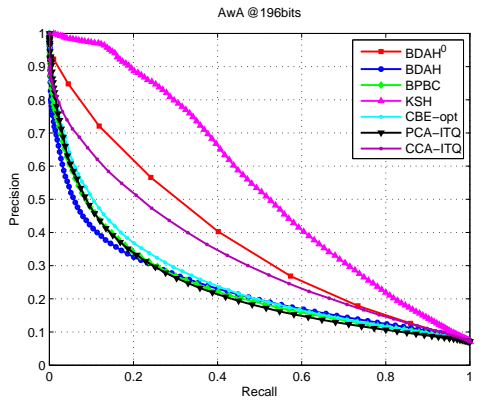

(d) P-R curves @196-bits

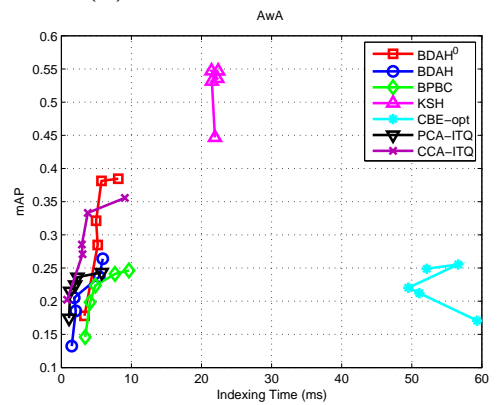

(f) Coding time vs. mAP for different bits code.

Fig. 2. P-R curves and mAP vs time for different methods with experiments on the AwA dataset. 


\begin{tabular}{|c|c|r|r|r|r|r|}
\hline \multicolumn{2}{|c|}{ Methods } & $\mathbf{1 6 b i t s}$ & $\mathbf{2 5 b i t s}$ & $\mathbf{6 4 b i t s}$ & $\mathbf{1 0 0 b i t s}$ & $\mathbf{2 5 6}$ bits \\
\hline \multirow{2}{*}{ BDAH $^{0}$} & Training & 46043.92 & 68347.63 & 69742.61 & 70820.15 & 79177.46 \\
\cline { 2 - 8 } & Coding & 3.30 & 5.19 & 4.95 & 5.72 & 8.12 \\
\hline \multirow{2}{*}{ BDAH } & Training & 43357.59 & 45544.10 & 46823.71 & 69739.86 & 75129.19 \\
\cline { 2 - 8 } & Coding & 1.48 & 2.05 & 1.83 & 5.42 & 5.91 \\
\hline \multirow{2}{*}{ BPBC } & Training & 19041.92 & 22239.26 & 23627.65 & 59662.54 & 65745.51 \\
\cline { 2 - 8 } & Coding & 3.40 & 4.09 & 4.85 & 7.65 & 9.64 \\
\hline \multirow{2}{*}{ KSH } & Training & 33409.12 & 73810.13 & 132546.38 & 20771.29 & 524378.30 \\
\cline { 2 - 8 } & Coding & 21.91 & 21.48 & 22.25 & 21.43 & 22.39 \\
\hline \multirow{2}{*}{ CBE-opt } & Training & 255049.87 & 255402.27 & 254791.00 & 258713.2595 & 253481.55 \\
\cline { 2 - 8 } & Coding & 59.35 & 51.08 & 49.52 & 56.63 & 52.14 \\
\hline \multirow{2}{*}{ PCA-ITQ } & Training & 3650.91 & 4479.65 & 5570.59 & 6892.80 & 16345.93 \\
\cline { 2 - 7 } & Coding & 1.10 & 1.25 & 1.96 & 2.30 & 5.75 \\
\hline \multirow{2}{*}{ CCA-ITQ } & Training & 20432.65 & 20912.83 & 21528.06 & 23202.85 & 32025.13 \\
\cline { 2 - 7 } & Coding & 0.82 & 3.01 & 2.92 & 3.78 & 9.06 \\
\hline
\end{tabular}

Table 3. Total training time (milliseconds) of different methods on the AWA dataset.

we randomly chose 50 classes, and picked 100 images per class as the training set, and 10 image per class as the test set. On the chosen data in ILSVRC2010, we utilized the publicly available SIFT features, clustered them into 500 centers and calculated 64000-dimensional VLAD features of each image. In our experiments, we used 64000-dimensional VLAD feature vectors to calculate hash codes.

\begin{tabular}{|c|c|c|c|c|c|c|c|c|}
\hline Methods & \multicolumn{2}{|c|}{ BDAH $^{0}$} & \multicolumn{2}{c|}{ BDAH } & \multicolumn{2}{c|}{ BPBC } & \multicolumn{2}{c|}{ KSH } \\
\hline bit & Training & Coding & Training & Coding & Training & Coding & Training & Coding \\
\hline 16bits & 488.74 & 0.32 & 482.98 & 0.31 & 151.18 & 0.35 & 39.65 & 0.87 \\
64bits & 507.35 & 0.34 & 494.52 & 0.33 & 163.51 & 0.36 & 138.60 & 0.89 \\
256bits & 543.44 & 0.41 & 514.64 & 0.36 & 182.84 & 0.39 & 553.51 & 0.90 \\
1024bits & 611.91 & 0.48 & 579.24 & 0.40 & 237.09 & 0.43 & 2193.67 & 0.93 \\
\hline
\end{tabular}

Table 4. Time consumption (seconds) of different methods on the ILSVRC2010 dataset.

We set the hash code length to 16bits, $64 \mathrm{bits}$ and $256 \mathrm{bits}$ and 1024bits on this dataset. CCA-ITQ and PCA-ITQ need to calculate the covariance matrix of the dataset. The 64000 dimension of this dataset is too high that our storage cannot contain the covariance matrix. So we did not compare with these two methods in this experiment. The results of retrieval on ILSVRC2010 are reported in Figure 3. Compared to supervised methods, our $\mathrm{BDAH}^{0}$ indexes more quickly than $\mathrm{KSH}$. Compared to unsupervised methods, $\mathrm{BDAH}^{0}$ performs better than $\mathrm{BPBC}$ in accuracy but consumes comparable time and memory as BPBC. Table 4 shows the specific training time and coding time of each method. 


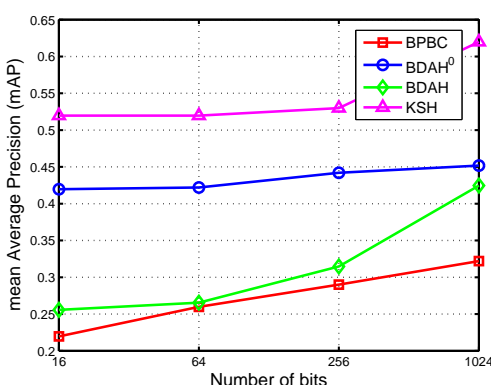

(a) mAP for different bits code.

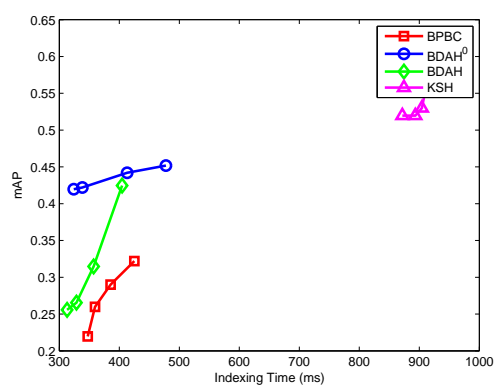

(b) Coding time vs. mAP for different bits code.

Fig. 3. Results on the ILSVRC2010 dataset.

\section{Conclusions}

In this paper, we have introduced a supervised hashing scheme with bilinear projection. With bilinear projection, our method can better deal with highdimensional dataset with less time and memory consumption than traditional supervised hashing schemes. Furthermore, our method processes input data in $2 \mathrm{D}$ form, thus it is very convenient to index 2-D structure raw-images and features. The experiments show that our method exceeds other unsupervised hashing schemes for high-dimensional data in accuracy. Moreover, our hashing method achieves comparable accuracy than other supervised methods while occupying much less memory and time.

Acknowledgement. This work was supported by NSFC project No.61370123 and BNSF project No.4162037.

\section{References}

1. Kulis, B., Darrell, T.: Learning to hash with binary reconstructive embeddings. In: NIPS. (2009) 1042-1050

2. Wang, J., Kumar, S., Chang, S.F.: Semi-supervised hashing for scalable image retrieval. In: CVPR, IEEE (2010) 3424-3431

3. Norouzi, M., Blei, D.M.: Minimal loss hashing for compact binary codes. In: ICML. (2011) 353-360

4. Strecha, C., Bronstein, A.M., Bronstein, M.M., Fua, P.: Ldahash: Improved matching with smaller descriptors. TPAMI 34 (2012) 66-78

5. Liu, W., Wang, J., Ji, R., Jiang, Y.G., Chang, S.F.: Supervised hashing with kernels. In: CVPR. (2012) 2074-2081

6. Shen, F., Shen, C., Liu, W., Tao Shen, H.: Supervised discrete hashing. In: CVPR. (2015) 37-45

7. Yang, H., Bai, X., Liu, Y., Wang, Y., Bai, L., Zhou, J., Tang, W.: Maximum margin hashing with supervised information. Multimedia Tools \& Applications $\mathbf{7 5}$ (2016) 3955-3971 
8. Andoni, A., Indyk, P.: Near-optimal hashing algorithms for approximate nearest neighbor in high dimensions. In: Foundations of Computer Science, 2006. FOCS'06. 47th Annual IEEE Symposium on, IEEE (2006) 459-468

9. Weiss, Y., Torralba, A., Fergus, R.: Spectral hashing. In: NIPS. (2008) 1753-1760

10. Liu, W., Wang, J., Kumar, S., Chang, S.F.: Hashing with graphs. In: ICML. (2011) $1-8$

11. Gong, Y., Lazebnik, S., Gordo, A., Perronnin, F.: Iterative quantization: A procrustean approach to learning binary codes for large-scale image retrieval. TPAMI 35 (2013) 2916-2929

12. Kulis, B., Grauman, K.: Kernelized locality-sensitive hashing. TPAMI 34 (2012) 1092-1104

13. Heo, J.P., Lee, Y., He, J., Chang, S.F., Yoon, S.E.: Spherical hashing. In: CVPR. (2012) 2957-2964

14. Yang, H., Bai, X., Zhou, J., Ren, P., Zhang, Z., Cheng, J.: Adaptive object retrieval with kernel reconstructive hashing. In: CVPR. (2014) 1955-1962

15. Bai, X., Yang, H., Zhou, J., Ren, P., Cheng, J.: Data-dependent hashing based on p-stable distribution. TIP 23 (2014) 5033-46

16. Xiao, B., Hancock, E.R., Wilson, R.C.: Graph characteristics from the heat kernel trace. Pattern Recognition 42 (2010) 2589-2606

17. Zhang, H., Bai, X., Zhou, J., Cheng, J., Zhao, H.: Object detection via structural feature selection and shape model. TIP 22 (2013) 4984-4995

18. Perronnin, F., Dance, C.: Fisher kernels on visual vocabularies for image categorization. In: CVPR. (2007) 1-8

19. Jgou, H., Douze, M., Schmid, C., Prez, P.: Aggregating local descriptors into a compact image representation. In: CVPR. (2010) 3304-3311

20. Jia, Y., Shelhamer, E., Donahue, J., Karayev, S., Long, J., Girshick, R., Guadarrama, S., Darrell, T.: Caffe: Convolutional architecture for fast feature embedding. arXiv preprint arXiv:1408.5093 (2014)

21. Gong, Y., Kumar, S., Rowley, H.A., Lazebnik, S.: Learning binary codes for highdimensional data using bilinear projections. In: CVPR. (2013) 484-491

22. Yu, F.X., Kumar, S., Gong, Y., Chang, S.F.: Circulant binary embedding. In: ICML. (2014) 946-954

23. Yan, X., Kaiming, H., Pushmeet, K., Jian, S.: Sparse projections for highdimensional binary codes. In: CVPR. (2015) 3332-3339

24. Ye, J., Janardan, R., Li, Q.: Two-dimensional linear discriminant analysis. In: NIPS. (2004) 1569-1576

25. Jinjun, W., Jianchao, Y., Kai, Y., Fengjun, L., Thomas, H., Yihong, G.: Localityconstrained linear coding for image classification. In: CVPR. (2010) 3360-3367

26. Lampert, C.H., Nickisch, H., Harmeling, S.: Learning to detect unseen object classes by between-class attribute transfer. In: CVPR, IEEE (2009) 951-958

27. Lécun, Y., Bottou, L., Bengio, Y., Haffner, P.: Gradient-based learning applied to document recognition. Proceedings of the IEEE 86 (1998) 2278-2324

28. Deng, J., Dong, W., Socher, R., Li, L.J., Li, K., Li, F.F.: Imagenet: A large-scale hierarchical image database. In: CVPR. (2009) 248-255

29. Fisher, R.A., et al.: The use of multiple measurements in taxonomic problems. Annals of Eugenics 7 (1936) 179-188

30. Schönemann, P.H.: A generalized solution of the orthogonal procrustes problem. Psychometrika 31 (1966) 1-10

31. Krizhevsky, A., Hinton, G.: Learning multiple layers of features from tiny images. Master's thesis, Department of Computer Science, University of Toronto (2009)

32. Leacock, C., Chodorow, M.: WordNet: An electronic lexical database. (1998) 